\title{
Cataclysmic Variables and Related Objects
}

\section{Proceedings of the 158th Colloquium of the International Astronomical Union, held at Keele, United Kingdom, June 26-30, 1995}

\section{A. EVANS AND JANET H. WOOD (EDS.)}

These proceedings present reviews and new results in the observational and theoretical study of cataclysmic variables (CVs) and related objects. A broad range of topics is included and there are thirteen reviews, covering magnetic and non-magnetic systems, accretion discs, observations of CVs from space, novae and symbiotic stars, $X$-ray binaries, supernovae and supersoft $X$-ray sources, and post common envelope binaries and evolution. There is a comprehensive subject and object index.

\section{Audience}

Astronomers and astrophysicists. 